\title{
Evaluation of Facility Effects on Ion Migration in a Hall Thruster Plume
}

\author{
Daniel L. Brown* \\ U.S. Air Force Research Laboratory, Edwards Air Force Base, California 93524 \\ and \\ Alec D. Gallimore \\ University of Michigan, Ann Arbor, Michigan 48109 \\ DOI: $\underline{10.2514 / 1 . B 34068}$
}

\begin{abstract}
A nested Faraday probe was designed and fabricated to assess facility effects in a systematic study of ion migration in a Hall thruster plume. Angular distributions of ion current density were studied at 8, 12, 16, and 20 thruster diameters downstream of a Hall effect thruster with four Faraday probe configurations at background pressures of $3 \times 10^{-6}, 1 \times 10^{-5}$, and $3 \times 10^{-5}$ torr. The effects of background facility neutrals were characterized and isolated, which enabled precise and accurate estimation of thruster ion beam current and plume divergence. A set of guidelines are recommended for Faraday probe design, experimental methodology, and data analysis that are aimed at minimizing uncertainty of far-field Faraday probe measurements. These guidelines were shown to reduce the experimentally derived ion beam current by 10-20\%, compared with conventional analysis techniques, and to reduce measurement uncertainty to approximately $\pm 3 \%$. The reductions in measurement uncertainty and the increased capability to approximate the onorbit plume expansion from ground-based measurements are significant improvements that can be used for validation of numerical simulations and investigations of Hall thruster performance loss mechanisms.
\end{abstract}

\section{Nomenclature}

$A_{C} \quad=$ cross-sectional geometric area of Faraday probe collector

$A_{0,1,2} \quad=$ second-order polynomial coefficients

$G_{0,1,2,3,4}=$ Gaussian function coefficients

$I=$ measured ion current in the plume at $[\theta, R, p]$

$I_{\text {Axial }}=$ axial component of ion beam current parallel to thrust axis

$I_{\text {Beam }} \quad=$ ion beam current

$I_{d} \quad=$ thruster discharge current

$I_{\text {Stripe }}=$ ion beam current passing through a constant angular width stripe

$p \quad=$ calculated facility background pressure

$p_{b} \quad=$ facility base pressure for air

$p_{i} \quad=$ pressure recorded from gauge

$R=$ downstream measurement distance from axis of rotation

$R_{\mathrm{CL}} \quad=$ thruster channel centerline radius

$R_{L}, R_{R} \quad=$ downstream distance from left or right ion point source to probe

$\alpha_{A}=$ ion angle of divergence relative to channel centerline

$\alpha_{L}, \alpha_{R}=$ angle of incidence from left and right ion point sources to probe

$\theta=$ angular position, horizontal plane in spherical coordinate system, $90^{\circ}$ on thrust axis
Received 12 July 2010; revision received 20 December 2010; accepted for publication 29 December 2010. This material is declared a work of the U.S. Government and is not subject to copyright protection in the United States. Copies of this paper may be made for personal or internal use, on condition that the copier pay the $\$ 10.00$ per-copy fee to the Copyright Clearance Center, Inc., 222 Rosewood Drive, Danvers, MA 01923; include the code 0748-4658/ 11 and $\$ 10.00$ in correspondence with the CCC.

*Program Manager, High Power Electric Propulsion; Spacecraft Branch, Propulsion Directorate; daniel.brown@edwards.af.mil. Associate Member AIAA.

${ }^{\dagger}$ Laboratory Director, Plasmadynamics and Electric Propulsion Laboratory; Professor, Department of Aerospace Engineering; Associate Dean, Horace H. Rackham School of Graduate Studies; alec.gallimore@ umich.edu. Fellow AIAA.

$$
\begin{aligned}
& \kappa_{A}=\text { correction accounting for ion angle of incidence } \\
& \text { to probe } \\
& \kappa_{D} \quad=\text { correction accounting for probe distance to channel } \\
& \kappa_{G}=\text { correction accounting for ion collection in Faraday } \\
& \text { probe gap } \\
& \lambda=\text { plume momentum divergence half-angle, } 0^{\circ} \text { on } \\
& \text { thrust axis } \\
& \tau=\text { change in ion current density with change in facility } \\
& \text { background pressure } \\
& \langle\rangle_{J}=\text { current-weighted average quantity in plume at } \\
& \text { constant } R(0<\theta<\pi) \\
& \langle\rangle_{m v}=\text { momentum-weighted average quantity in plume at } \\
& \text { constant } R(0<\theta<\pi)
\end{aligned}
$$

\section{Introduction}

$\mathbf{H}$ ALL thrusters operate through the plasmadynamic interactions of applied electric and magnetic fields with injected propellant and electrons from a cathode. The advantages of Hall effect thruster (HET) technology for rapid high velocity increment Earth-centric maneuvers and interplanetary trajectories are due to high-efficiency operation and high thrust density for specific impulse in the range of 1000 to $5000 \mathrm{~s}$. Knowledge of ion current density in the plasma beam may be used to quantify dominant performance loss mechanisms associated with plume divergence, the ionization mass fraction, and electron current to the anode. Faraday probes have been used to quantify these characteristics in numerous laboratory investigations, but they have a high degree of uncertainty attributed to facility effects. These facility interactions have been investigated by many groups with several Hall thruster designs, all of which found that facility effects escalate with background pressure and may alter thruster performance and plume properties [1-5]. The consequences of these interactions on the SPT- 100 plume were clearly demonstrated by comparisons of ground measurements with onorbit ion current density data from the Express communication satellite, which revealed significant differences in ion density magnitude and plume collimation [6-8].

Facility effects associated with background neutral particles that are inherent in ground-based Hall thruster investigations manifest in two ways. The first is ionization and subsequent acceleration of 
facility neutral particles near the discharge exit, which results in a population of low-velocity highly divergent ions that increase thruster discharge current, thrust, and measured ion current density in the plume. The second interaction arises through charge-exchange (CEX) collisional processes between ions generated in the thruster discharge channel with background facility neutral particles, which results in particle scattering that increases current density on the periphery of the plume.

A number of investigations have evaluated nude Faraday probe design modifications to minimize collection of low-energy ions generated through facility effects. The modifications include filtering mechanisms designed to mitigate the collection of low-energy ions in electric propulsion plumes. One approach is to attach a collimator to the entrance of a nude Faraday probe to attenuate dispersed lowenergy ions that are created by CEX collisions $[3,9]$. Other Faraday diagnostics use electric or magnetic fields to filter low-energy CEX ions. A study by Rovey et al. [10] compared results from a magnetically filtered Faraday probe, a boxed Faraday probe, and a nude Faraday probe to separately assess the effect of the magnetic filter and the boxed collimator that houses the filter. The magnetically filtered and boxed Faraday probes recorded reduced ion beam current and diminished current density on the plume periphery compared with the uncollimated Faraday probe. These findings indicate the boxed collimator and magnetic filtering attenuated the low-energy CEX ions collected by the Faraday probe. The disadvantage of collimator and filtering configurations are that they do not selectively isolate facility effects from the ionization of thruster and cathode neutrals downstream of the primary acceleration zone. A collimated Faraday probe collects both the low-energy thruster ion population and CEX facility ions created near the thruster exit, whereas the magnetically filtered Faraday probe does not collect either population. Therefore, neither diagnostic accurately measures the onorbit plume characteristics of an electric propulsion system.

Several analytical methods have been developed to account for collection of low-energy facility CEX ions in the periphery of the plume. Nevertheless, the integrated ion beam current from far-field measurements is typically larger than the value reported from nearfield measurements, and it is often greater than the thruster discharge current. Analytical techniques include subtracting the ion current density at the periphery of the plume $\left(\theta=0\right.$ and $\left.180^{\circ}\right)$ from the entire beam profile or extrapolating the exponential region $\left(30^{\circ}<\theta<60^{\circ}\right.$ and $120^{\circ}<\theta<150^{\circ}$ ) of the ion current density to the outer periphery $\left(0^{\circ}<\theta<30^{\circ}\right.$ and $\left.150^{\circ}<\theta<180^{\circ}\right)$. While these approaches provide a simple alternative to the experimental methods, they are limited in determining the spatial influence of facility effects throughout the plume. Subtracting a finite current density from the profile is based on the assumption that probe collection of ambient facility ions is uniform throughout the plume. The exponential extrapolation technique is based on the spatial decay of beam ions on the edges of the primary beam, but it removes features of the outer periphery that may arise due to CEX collisions near the thruster exit. Neither of these techniques accurately captures the angular distribution of low-energy ions that would be present on orbit.

To further study the overprediction of integrated ion beam current, plasma potential in the region surrounding a nude Faraday probe was measured to study the possibility of probe bias voltage acting as a point source potential sink, and thereby attracting low-energy CEX ions [11]. Langmuir probe measurements near a nude Faraday probe resulted in potential variations less than $3 \mathrm{~V}$ within $20 \mathrm{~mm}$ of the probe. It was concluded that CEX ions had a negligible attraction to the negative probe bias potential, and the random flux of low-energy ions was insufficient to explain overpredicted ion beam current compared with thruster discharge current. This conclusion was consistent with a hybrid-particle-in-cell (PIC) model of the ion flow around an axisymmetric Faraday probe, which concluded ion collection errors due to sheath expansion were minimal [12].

A different method for discerning CEX processes in the plume was demonstrated by de Grys et al. [3] and Azziz [13], who compared Faraday probe measurements at individual locations in the plume at several background pressures and extrapolated the current density to vacuum conditions. This approach isolates the influence of background particles from the interactions between thruster ions and neutral propellant, which is a primary advantage over Faraday probe designs that filter low-energy ions. The ability to experimentally assess facility effects in the plume at discrete locations enables a more accurate means of characterizing onorbit ion current density profiles.

Despite extensive study of experimental methods and analytical techniques, Faraday probe measurements continue to be complicated by facility effects. In a past study, a Faraday probe with nested, concentric collectors was developed to assess the effect of probe design and geometry on the ion collection area [14]. The study identified ion current collection by the sidewall of the collector, which increased the calculated current density when the crosssectional collector area was used. A correction factor was proposed to adjust the effective probe collection area to account for ions collected by the sidewalls of the collector and guard ring, which decreased the ion current density by 10 to $20 \%$ and resulted in values of integrated ion beam current consistent with expected values based on Hall thruster performance analyses [14]. The correction factor was applied to all measurements in this investigation.

The primary aim of this study was to systematically evaluate a Hall thruster plume in order to isolate facility effects and characterize ion migration in the space environment. The influence of vacuum test facilities on measurements of collected ion current in the plume of a low-power Hall thruster was characterized by adjusting facility background pressure and measurement distance from the thruster exit plane. Facility effects attributed to background neutral gas were evaluated using methods analogous to de Grys et al. [3] and Azziz [13]. This method was evaluated with four nested Faraday probe configurations, and consistent agreement in measured plume properties between the four configurations was considered an accurate means to isolate facility effects and ascertain the influence of CEX collisions on ion migration in a Hall thruster plume. Because of the large spatial region surveyed in this investigation, an analytical study of systematic measurement error associated with using a spherical measurement coordinate system for a thruster with annular geometry is included. Findings were combined with past recommendations to generate guidelines for Faraday probe design, experimental methods, and analysis of results. Results of this investigation indicate these guidelines will reduce Faraday probe measurement uncertainty to approximately $\pm 3 \%$, which is significant improvement over the estimated $\pm 20 \%$ or greater that is commonly reported. The results enable quantitative rather than qualitative evaluation of ion current density in the Hall thruster plume and detailed mapping of facility effects on ion migration for determination of onorbit plume characteristics.

\section{Experimental Apparatus}

\section{A. Vacuum Facility and Hall Thruster Ion Source}

The evaluation of facility effects and ion migration was conducted in chamber 1 at the U.S. Air Force Research Laboratory (AFRL). Chamber 1 is a stainless steel, cylindrical vacuum chamber $2.3 \mathrm{~m}$ in diameter and $4 \mathrm{~m}$ in length. A schematic is shown in Fig. 1. A low-power Hall thruster was located along the centerline of the chamber and fired toward the cryopanels at the opposing end. All surfaces exposed to direct impingement by the plasma plume were shielded with graphite felt. An Edwards DryStar GV160 mechanical booster pump was used to reach rough vacuum, and lighter gases were removed with a Varian TV55 turbomolecular pump. Two CVi TorrMaster cryotubs circulated liquid nitrogen to cool four cryopanels, and they achieved a measured xenon pumping speed of $50,000 \mathrm{l} / \mathrm{s}$.

An MKS Instruments HPS-series cold cathode ionization gauge (CCG) was located on the chamber ceiling above the thruster centerline approximately $1 \mathrm{~m}$ downstream of the exit plane. Facility background pressure $p$ was calculated with a xenon correction factor of 2.87 using Eq. (1):

$$
p=\frac{p_{i}-p_{b}}{2.87}+p_{b}
$$




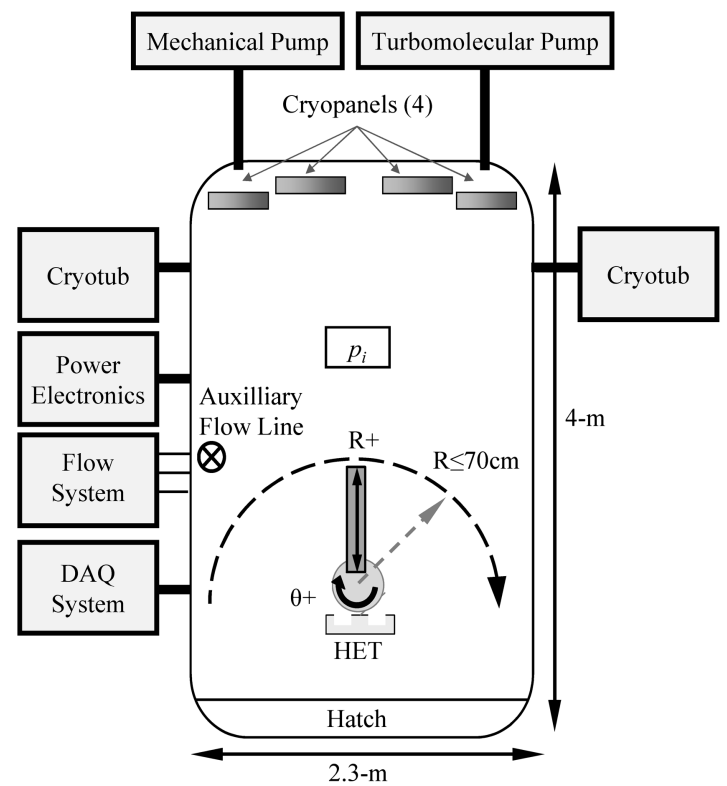

Fig. 1 Schematic of chamber 1 at AFRL (not to scale).

where $p_{i}$ is the pressure measured by the ionization gauge and $p_{b}$ is the base pressure of air, which was $p_{b} \approx 1 \times 10^{-7}$ torr. Chamber background pressure was increased by injecting xenon through an auxiliary flow line located approximately $1 \mathrm{~m}$ downstream of the thruster exit plane. Injected flows of approximately 9, 29, and $127 \mathrm{sccm}$ corresponded to corrected xenon background pressures of $3.2 \times 10^{-6}, 1.1 \times 10^{-5}$, and $3.5 \times 10^{-5}$ torr, respectively. According to MKS Instruments, the CCG measurement reproducibility is within $5 \%$ of reading at constant temperature [15]. Uncertainty of the CCG was estimated at $\pm 20 \%$.

The diagnostic positioning system consisted of a translation stage for control of measurement radius and a rotation stage, which was centered beneath the exit plane on thruster centerline. One end of the translation stage was mounted on top of the rotation stage, enabling current density scans from 0 to $180^{\circ}$ at constant radius from the axis of rotation at the exit plane. Far-field measurements were taken in $2^{\circ}$ increments for an axisymmetric, spherical coordinate geometry. The Faraday probe and thruster were mounted approximately $50 \mathrm{~cm}$ above the rotation and translation stages. The overall uncertainty in measurement position was estimated at $\pm 0.5 \mathrm{~cm}$.

\section{B. Nested Faraday Probe}

A nested Faraday probe with two concentric collectors and an outer guard ring was used in this investigation to enable simultaneous measurements of ion current density in the Hall thruster plume with the inner and outer collectors. The inner collector was $6 \mathrm{~mm}$ diameter, and two versions of the outer collector were machined to create a gap of either 0.5 or $1.5 \mathrm{~mm}$ between the rings. One version of the outer collector was machined to a $\sim 7 \mathrm{~mm}$ inner diameter and $\sim 19 \mathrm{~mm}$ outer diameter to create $0.5 \mathrm{~mm}$ gaps between the inner and outer collectors and between the outer collector and guard ring. A second version of the outer collector was machined to a $\sim 9 \mathrm{~mm}$ inner diameter and $\sim 17 \mathrm{~mm}$ outer diameter to create $1.5 \mathrm{~mm}$ gaps between the inner and outer collectors and between the outer collector and guard ring. The $0.5 \mathrm{~mm}$ gap configuration is shown in Fig. 2 .

Collected ion current was measured with both versions of the outer collector, which resulted in four probe collection geometries that were studied using the nested Faraday probe. These configurations are defined as 1) configuration 1: the current to the inner collector with a $0.5 \mathrm{~mm}$ gap, 2) configuration 2: the combined current to the inner and outer collectors with a $0.5 \mathrm{~mm}$ gap, 3 ) configuration 3 : the current to the inner collector with a $1.5 \mathrm{~mm}$ gap, and 4) configuration 4: the combined current to the inner and outer collectors with a $1.5 \mathrm{~mm}$ gap.

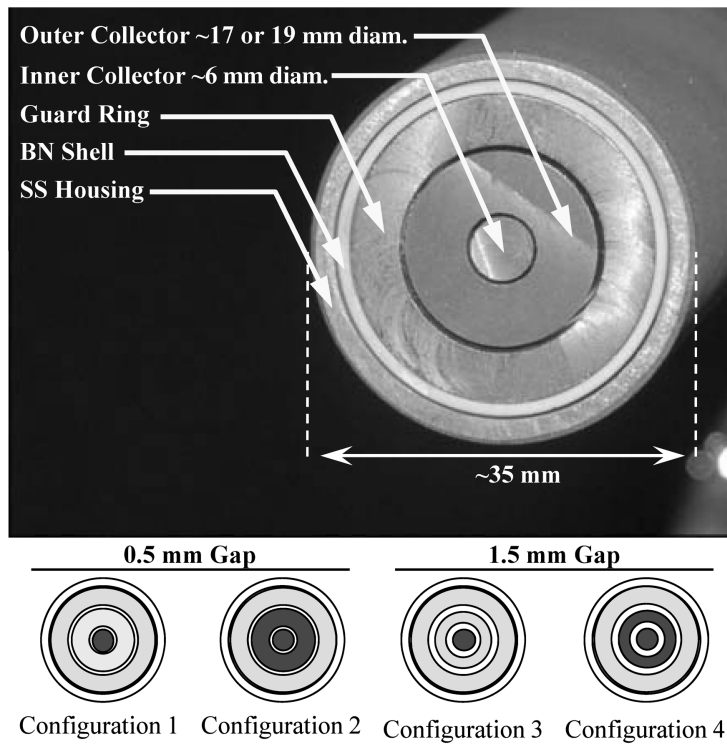

Fig. 2 Photograph of the AFRL nested Faraday probe shown in the $0.5 \mathrm{~mm}$ gap width configuration. Diagrams of the four probe configurations are shown for the 0.5 and $1.5 \mathrm{~mm}$ gap geometries.

Collected current was measured with an Agilent 34970A data acquisition (DAQ)/switch unit, as illustrated in Fig. 3. An Agilent E3631A triple-output dc power supply was used to bias the collectors and guard ring. The nested Faraday probe operation was characterized with variation in probe bias potential over a range of angular positions and downstream distances at several facility background pressures. A bias potential of $-20 \mathrm{~V}$ with respect to facility ground was beyond the ion current saturation limit in all cases, and it was used for all Faraday probe measurements. Current to the inner and outer collectors was recorded simultaneously. Separate thruster firings were required to allow testing with each of the different versions of the outer collector.

The nested Faraday probe collectors were machined from arc-cast low-carbon grade-365 molybdenum. The guard ring was grade-360 molybdenum. Differences in secondary electron emission (SEE) between the collectors and guard ring was assumed negligible. A boron nitride (BN) shell surrounded the guard ring, and stainless steel (SS) housing enclosed the probe. This housing was electrically grounded to the chamber. Additional details on the facility, nested Faraday probe configurations, and experimental methods were published elsewhere [14].

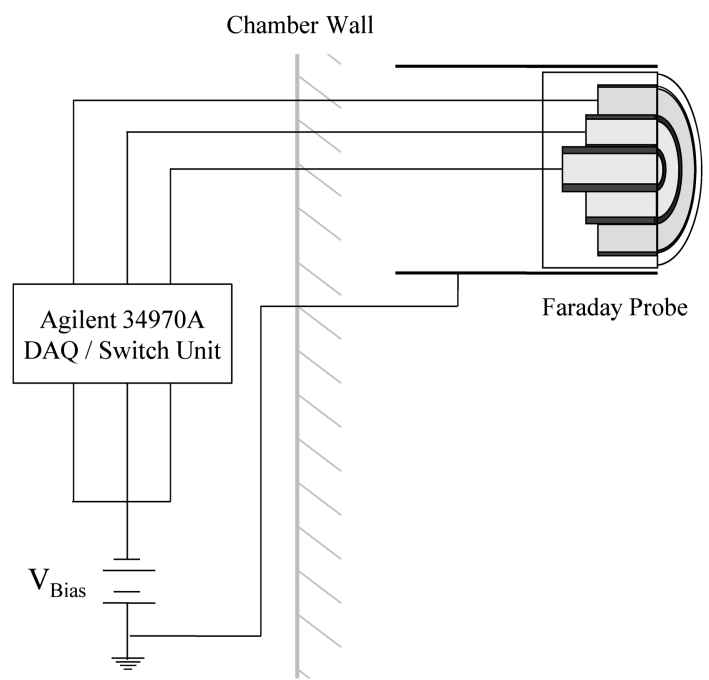

Fig. 3 Electrical diagram of nested Faraday probe power electronics and DAQ system. 


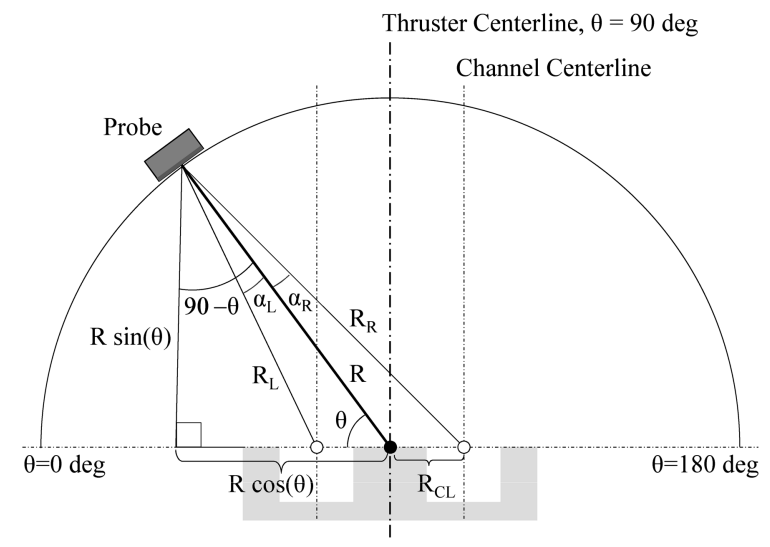

Fig. 4 Measurement coordinate system showing probe distance and angular location in a two-point source model.

\section{Analysis of Systematic Measurement Error}

Consideration of the measurement coordinate system was undertaken to isolate systematic trends due to facility effects in experimental measurements. The analysis was aimed at resolving the systematic error caused by probe measurements with respect to a point source as opposed to the annular discharge geometry of a typical HET. In this analysis, the thruster was modeled as two point ion sources located at the centerline of the discharge channel. Figure 4 illustrates the probe distance $R$ and angular location $\theta$ with respect to the thruster centerline.

Two geometric corrections were analyzed. The first correction accounted for variations in probe angle with respect to the point sources, which affected the current collection area. In a single point source coordinate system, where the ion point source is located at the probe axis of rotation, the probe face is perpendicular to the source as it is swept in a $180^{\circ}$ arc. Modeling the thruster as two point sources changes this probe orientation, such that the probe face is only perpendicular to the point sources at 0 and $180^{\circ}$. The ion angle of incidence to the probe face changes with angular position and distance, and it decreases the effective probe collection area of beam ions. In addition, the ion angle of incidence at a given location is different for each point source. The angles of incidence were calculated for the left and right point sources as $\alpha_{L}$ and $\alpha_{R}$, and they were used to evaluate cosine losses in the probe collection area.

The angles $\alpha_{L}$ and $\alpha_{R}$ are formulated in Eq. (2) based on the geometry shown in Fig. $\underline{4}$, written as

$$
\alpha_{L, R}\left[\theta, R, R_{\mathrm{CL}}\right]= \pm\left[(\pi / 2)-\theta-\tan ^{-1}\left(\frac{\cos (\theta) \mp\left(R_{\mathrm{CL}} / R\right)}{\sin (\theta)}\right)\right]
$$

These angles are expressed in a generalized form based on $\theta, R$, and the channel centerline radius $R_{\mathrm{CL}}$. The ratio of $R / R_{\mathrm{CL}}$ is incorporated to simplify the analysis and enable a more direct comparison between large and small thrusters. The probe collection area was corrected for cosine losses with the area correction factor $\kappa_{A}$, using the average of $\alpha_{L}$ and $\alpha_{R}$ in Eq. (3):

$$
\kappa_{A}\left[\theta, R, R_{\mathrm{CL}}\right]=\cos ^{2}\left(\frac{\alpha_{L}+\alpha_{R}}{2}\right)
$$

The second correction accounts for differences in path length from the left and right point sources to the probe, which would introduce systematic error in the $R^{2}$ term in the axisymmetric plume integration. The probe distances from the left and right point sources are characterized as $R_{L}$ and $R_{R}$. Similar to the analysis of ion angle of incidence, the path length will vary with probe angular position and is dissimilar for each point source. The exception is on thruster centerline, where the distance from the probe to each point source is equal and greater than the measurement radius of rotation $R$.

The lengths $R_{L}$ and $R_{R}$ were calculated with respect to the measurement distance $R$ in Eq. (4):

$$
\frac{R_{L, R}\left[\theta, R, R_{\mathrm{CL}}\right]}{R}=\sqrt{[\sin (\theta)]^{2}+\left(\cos (\theta) \mp \frac{R_{\mathrm{CL}}}{R}\right)^{2}}
$$

The distance correction factor $\kappa_{D}$ is defined as a function of $\theta, R$, and $R_{\mathrm{CL}}$ in Eq. ( $)_{\text {): }}$

$$
\kappa_{D}\left[\theta, R, R_{\mathrm{CL}}\right]=\left[\frac{1}{2}\left(\frac{R_{L}}{R}+\frac{R_{R}}{R}\right)\right]^{2}
$$

The effects of $\kappa_{D}$ and $\kappa_{A}$ were applied to all Faraday probe current density measurements in this investigation, and the total ion beam current $I_{\text {Beam }}$ was calculated using Eq. (6) for a Faraday probe scan at constant measurement radius $R$ :

$$
I_{\text {Beam }}=2 \pi R^{2} \int_{0}^{\pi / 2} \frac{I[\theta, R]}{A_{C}+\kappa_{G}}\left(\frac{\kappa_{D}\left[\theta, R, R_{\mathrm{CL}}\right]}{\kappa_{A}\left[\theta, R, R_{\mathrm{CL}}\right]}\right) \sin [\theta+(\pi / 2)] \mathrm{d} \theta
$$

where $I[\theta, R]$ is the ion current measured by a Faraday probe at angular position $\theta$ and radius $R$. This formulation incorporates the gap correction factor $\kappa_{G}$ developed in a previous study with the nested Faraday probe [14]. The gap correction factor was applied to the geometric probe collection area $A_{C}$ to account for ions collected in the gap between the collector and guard ring. The correction increased the effective probe collection area by more than $10 \%$, and it resulted in consistent agreement in calculated current density between the four probe configurations with variations in collector size and gap width [14].

The ratio of spatial correction factors $\left(\kappa_{D} / \kappa_{A}\right)$ is displayed as a function of probe angular position in Fig. 5 in terms of a unit of measure specified as channel centerline diameters downstream (CCDD) of the diagnostic axis of rotation. The overall effect of this ratio was to increase current density in the plume central core, which ultimately increased the integrated ion beam current. Variation in collection area due to ion angle of incidence decreases rapidly with downstream distance, and the approximation of a point source measurement improves. In Fig. 6, the correction on thruster centerline is shown as a function of CCDD, calculated as $R / 2 R_{\mathrm{CL}}$. The overall correction factor asymptotically approaches unity with downstream measurement distance, and it is less than 1.01 for distances greater than $8 \mathrm{CCDD}$. Thus, including the spatial corrections minimizes a systematic source of error introduced from the hemispherical measurement system. All current density traces and beam current calculations in this investigation incorporated the spatial corrections for ion angle of incidence and measurement distance using the formulations in Eqs. (3), (5), and (6).

The corrections in probe collection area are only valid for beam ions originating near the exit plane. Charge exchange collisions

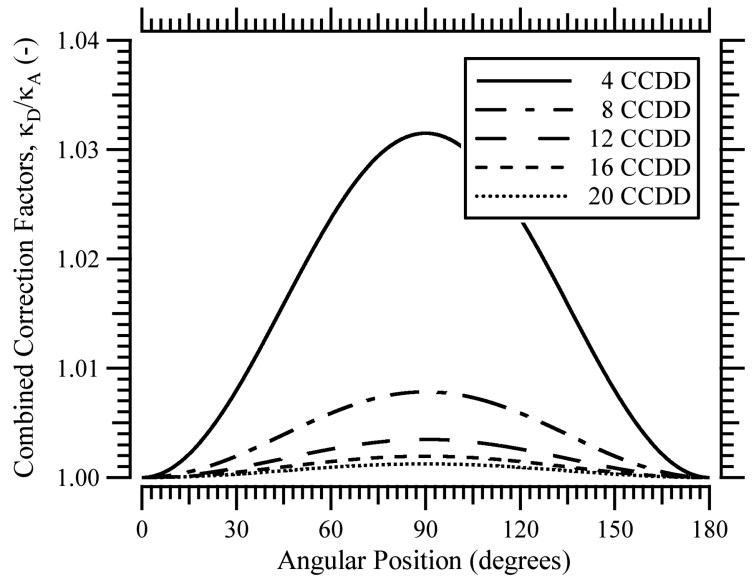

Fig. 5 Combined effect of correction factors $\left(\kappa_{D} / \kappa_{A}\right)$ accounting for probe distance and angle with respect to left and right ion point sources as a function of angular position with contours of constant $R / 2 R_{\mathrm{CL}}=4$, $8,12,16$, and 20 CCDD. 


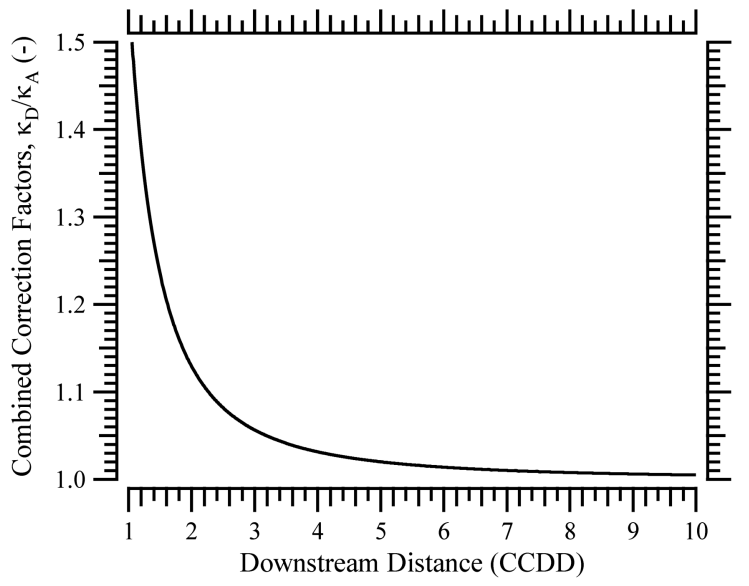

Fig. 6 Combined effect of the correction factors $\left(\kappa_{D} / \kappa_{A}\right)$ on channel centerline $\left(\theta=90^{\circ}\right)$ as a function of downstream distance (CCDD).

downstream of the primary ionization region increase dispersion of ion velocity, and the correction is not relevant to this population. In addition, the analysis does not account for channel width. This is of lesser concern, since minor variations in channel centerline radius will have a negligible effect on measurements taken beyond four thruster diameters.

\section{Results}

In this study of facility effects, ion current density was characterized throughout the plume as a function of background pressure. Using methods described by de Grys et al. [3] and Azziz [13], the ion current density was extrapolated to vacuum conditions at discrete angular locations to isolate effects arising from CEX collisions and ingestion of facility neutral particles. Results of this technique are shown in Fig. 7 for configuration 1 at 20 CCDD. Collected ion current was plotted in $10^{\circ}$ increments as a function of facility background pressure. On the thruster centerline at $\theta=90^{\circ}$, the collected current increased linearly with pressure. However, the slope of the collected ion current extrapolated to vacuum conditions $\tau$ transitioned at $\pm 10^{\circ}$ from the centerline and became slightly negative at \pm 20 and $\pm 30^{\circ}$ from the thruster centerline. The reverse trend occurred at approximately $\pm 40^{\circ}$ and resulted in a positive linear slope on the periphery from \pm 60 to $\pm 90^{\circ}$ from the thruster centerline.

Variations in the slope of collected ion current extrapolated to vacuum conditions $\tau$ in Fig. 7 are plotted as a function of angular position in Fig. 8 at 8, 12, 16, and 20 CCDD. Angular variation in the slope was analyzed to evaluate regions in the plume that were most sensitive to facility effects. Although profiles of angular current density often indicate the plume periphery is the region most affected by facility background pressure, angular variations in the slope reveal the central core is the region most influenced. This is revealed in Fig. $\underline{8}$, where the variation in collected ion current with background pressure was two orders of magnitude greater in the central core than the periphery.

Angular regions where $\tau$ transitioned from positive to negative occurred at approximately $\pm 10^{\circ}$ from thruster centerline for all downstream distances. Similarly, the transition from negative to positive slopes occurred at approximately $\pm 50^{\circ}$ from the thruster centerline for all downstream distances. Residuals in Fig. 8 quantified the validity of a linear relationship between changes in collected ion current with changes in facility background pressure at each measurement location, and they were calculated as the square of the Pearson product moment correlation coefficient. While the extrapolations to vacuum in this study were based on three data points at each angular location, the investigation by Azziz [13] also showed a linear relationship based on five data points over a reduced range of background pressure. Azziz reported residuals of greater than 0.99 throughout the plume and concluded this linear trend was valid at all locations. However, that study also found transition

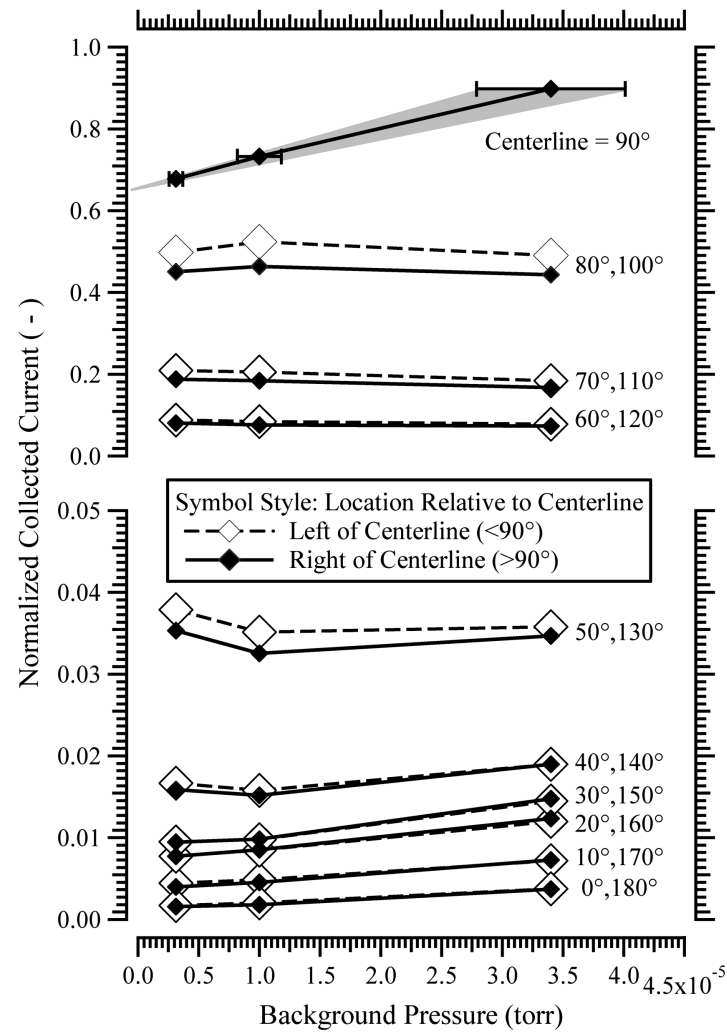

Fig. 7 Normalized collected ion current of configuration 1 as a function of background pressure at discrete angular locations in the plume at 20 CCDD. Measurements are normalized to the maximum collected current of the profile at $3.4 \times 10^{-5}$ torr.

regions between positive and negative slopes that were equivalent to those in Figs. 7 and $\underline{8}$. It is possible the data in [13] may have exhibited nonlinear relationships if data were taken in smaller angular increments and over a wider range of background pressure. Although the analysis in this investigation used the minimum number of data points for extrapolation to vacuum conditions, it was believed to have minimal effect on the vacuum ion current density

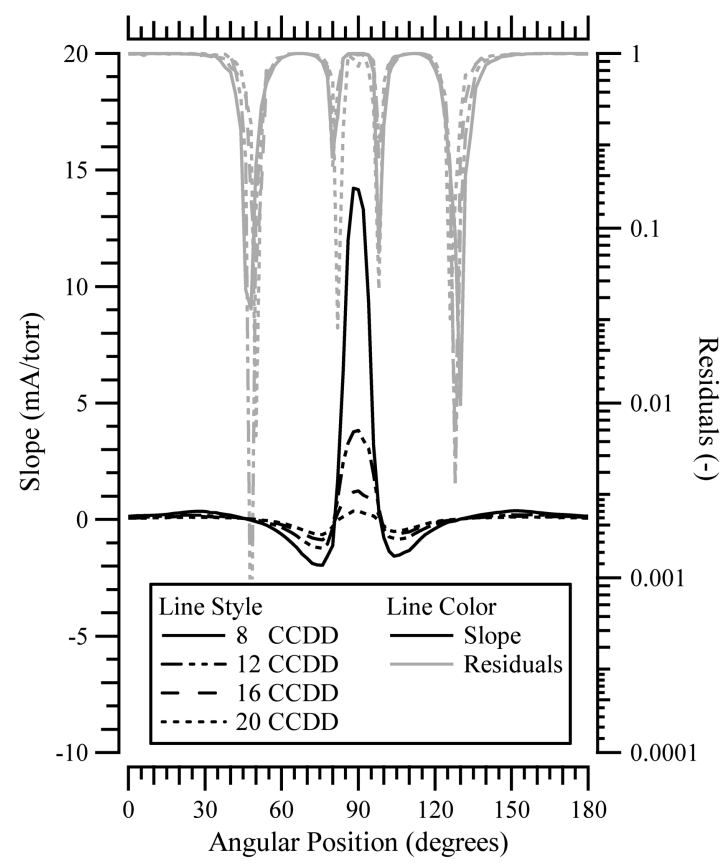

Fig. 8 Residuals and slope of collected ion current extrapolated to vacuum conditions of the nested Faraday probe configuration 1 as a function of angular position at $8,12,16$, and $20 \mathrm{CCDD}$. 
profiles for two reasons. In regions where residuals were near unity and $\tau$ exhibited a strong linear relationship with increasing pressure, additional data points were unlikely to change the slope. In addition, angular regions where the residuals declined were associated with a transition from positive to negative slopes, such that the slope was approximately zero and the variation in collected ion current density with facility background pressure was minimal.

One disadvantage of this technique was the uncertainty associated with determination of facility background pressure. This was due to both the measurement uncertainty of the CCG and the pressure gradients in the vacuum facility. A previous experimental investigation of the pressure gradient in the $5 \mathrm{~kW}$ P5 HET plume found minimal variation approximately $1.5 \mathrm{~m}$ downstream of the exit plane on the centerline [16]. This distance was approximately $10 \mathrm{CCDD}$ for the P5 (148 mm mean thruster diameter), which was comparable to the minimum distance of $8 \mathrm{CCDD}$ in this investigation. As a consequence of this result, the facility background pressure gradient was expected to be minimal for all distances from 8-20 CCDD.

The measurement uncertainty in CCG pressure readings was deemed to have a minor effect on the trends in Fig. 7. Although CCGs are known to exhibit a nonlinear relationship in the current-pressure characteristic, these attributes arise primarily at ultrahigh vacuum conditions and are attributed to gauge design, electrode operation, and field alignment [17]. The vacuum environment in this study ranged from $\sim 10^{-6}$ to $\sim 10^{-4}$ torr, where the CCG current-pressure characteristic has a higher degree of linearity [18]. Therefore, the CCG behavior was expected to be constant for all pressure measurements reported in this investigation. The $\pm 20 \%$ uncertainty in recorded pressure had minimal effect on the slope of ion current density extrapolated to vacuum conditions. This range in slope due to $\pm 20 \% x$-axis error bars is illustrated in Fig. 7 by the shaded region surrounding the centerline data. Although the centerline region had the largest slope and would be most affected by uncertainty in pressure, the extrapolation to vacuum conditions was insensitive to this uncertainty.

The slopes of the ion current density extrapolated to vacuum conditions in Fig. 8 were dominated by two distinct effects in the plume. These slopes were analyzed to extract information about the angular location of increased current density due to neutral ingestion and dispersion from beam scattering due to CEX collisions with facility neutrals. Neutral ingestion would increase ion current density in the central core and manifest in Fig. $\underline{8}$ as a positive slope on the thruster centerline at $\theta=90^{\circ}$. Beam scattering due to CEX collisions with facility neutrals would reduce ion current density in the central core and increase current density at the plume periphery. This scattering would appear as a negative slope in the central core in Fig. $\underline{8}$ and a positive slope on the plume periphery. The effects were each approximated with a Gaussian distribution, such that the angular distribution of slope $\tau$ was modeled as the superposition of the following Gaussian functions:

$$
\tau \cong G_{0}+G_{1} \exp \left[-\left(\frac{\theta-(\pi / 2)}{G_{2}}\right)^{2}\right]+G_{3} \exp \left[-\left(\frac{\theta-(\pi / 2)}{G_{4}}\right)^{2}\right]
$$

The influence of CEX collisions were modeled with Gaussian coefficients $G_{0}, G_{1}$, and $G_{2}$. The effects of facility neutral particle ingestion were modeled with $G_{3}$ and $G_{4}$. The individual Gaussian curves for ingestion and CEX collisions in the plume were determined with IGOR Pro curve-fitting to the experimental data. The angular distribution of $\tau$ from experimental data and Gaussian models are compared in Fig. 9 from 8 to 20 CCDD. The superposition of Gaussian curves showed consistent agreement with the measured angular distribution of slope for all downstream distances. Although the Gaussian function was subjective and may not be the correct physical distribution, this analysis provided a qualitative characterization of the angular range and relative magnitude of facility effects in the plume for this thruster configuration. Gaussian function constants $G_{0}, G_{1}, G_{2}, G_{3}$, and $G_{4}$ are listed in Table 1 for each downstream distance.

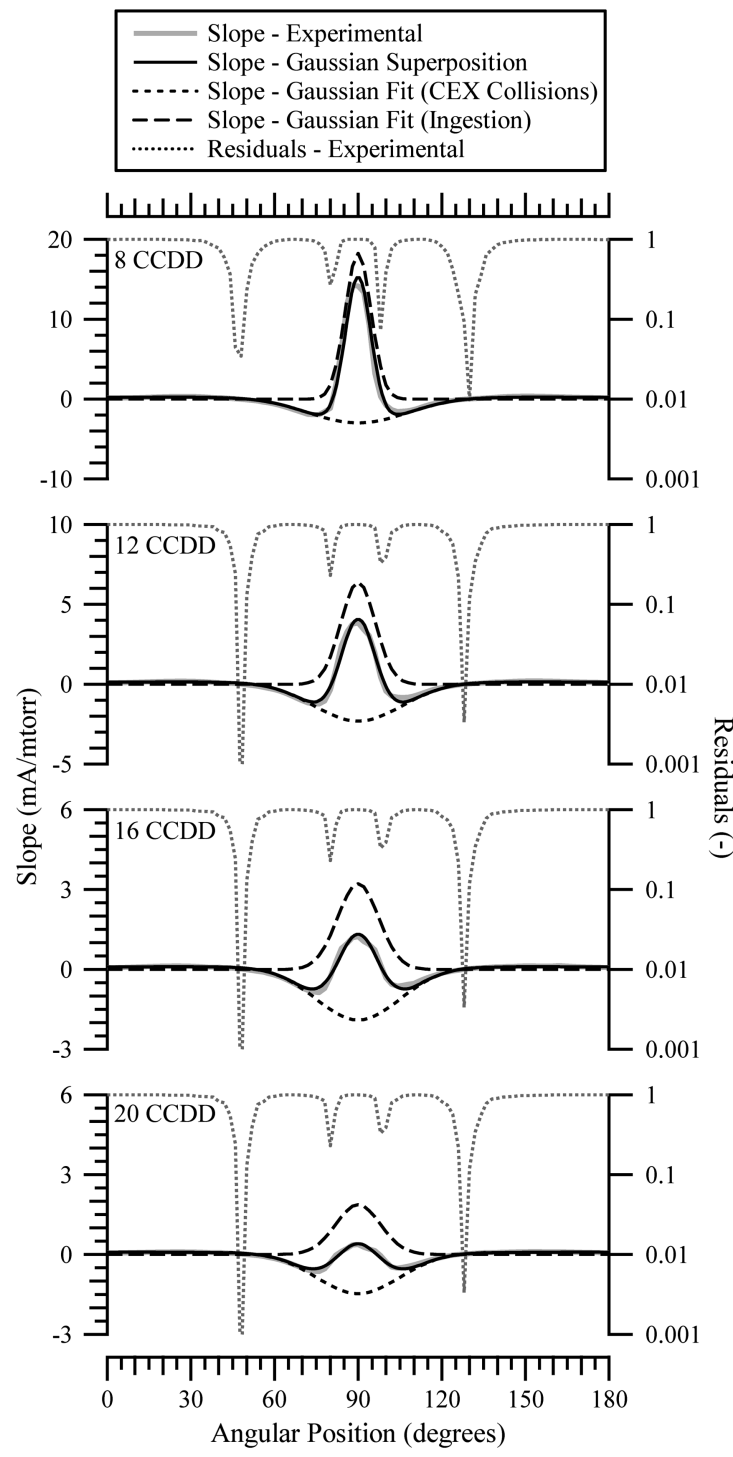

Fig. 9 Residuals and slope of collected ion current extrapolated to vacuum conditions as a function of angular position at $8,12,16$, and 20 CCDD. The experimental slope from configuration 1 is compared with the superposition of two Gaussian distributions at each downstream distance.

The effect attributed to ionization and acceleration of ingested facility neutrals led to a positive increase in slope about the thruster centerline. The magnitude of the positive slope in the central core decreased with downstream distance as approximately $R^{2}$. Ingestion affected a larger angular range of the plume as downstream distance increased, based on the increase in coefficient $G_{4}$ from 8 to 20 CCDD. In addition, ingestion of facility neutral particles caused a net increase in the integrated ion beam current, which corresponded to increased discharge current. The predicted increase in thruster discharge current calculated with the Gaussian distributions for ingested facility neutrals is plotted in Fig. 10 as a function of background pressure. These predictions match the measured thruster discharge current to within $0.02 \mathrm{~A}$ and exhibit better agreement at the furthest downstream distances.

The negative slope of ion current density extrapolated to vacuum was the result of CEX collisions with facility neutrals downstream of the primary acceleration zone, which influenced a broader angular region of the plume than ingestion and led to expansion of ions from the central core. The slope was positive beyond approximately $\pm 50^{\circ}$ from the thruster centerline for all downstream distances, and it was the source of increased ion current density on the periphery of the plume at elevated facility background pressure. The width of the Gaussian attributed to downstream CEX collisions with facility 
Table 1 Constants of best-fit Gaussian functions for neutral ingestion and CEX scattering as a function of downstream distance from 8 to $20 \mathrm{CCDD}$

\begin{tabular}{crrrrr}
\hline & \multicolumn{3}{c}{$\begin{array}{c}\text { Gaussian slope } \\
\text { CEX collisions }\end{array}$} & \multicolumn{2}{c}{$\begin{array}{c}\text { Gaussian slope } \\
\text { ingestion }\end{array}$} \\
\cline { 2 - 6 } $\begin{array}{c}\text { Downstream } \\
\text { distance, CCDD }\end{array}$ & $G_{0}$ & $G_{1}$ & $G_{2}$ & $G_{3}$ & $G_{4}$ \\
\hline 8 & 0.256 & -3.241 & 25.006 & 18.245 & 6.549 \\
12 & 0.144 & -2.447 & 22.435 & 6.377 & 8.723 \\
16 & 0.100 & -1.998 & 21.223 & 3.213 & 10.380 \\
20 & 0.079 & -1.546 & 21.231 & 1.868 & 11.339 \\
\hline \hline
\end{tabular}

neutrals decreased with downstream distance in the far-field plume. Ion current density profiles of the nested Faraday probe are evaluated in Figs. 11-13 over a range of measurement distances and facility background pressure. Profiles in Figs. 11-13 are normalized to the maximum ion current density of configuration 1 at vacuum conditions and 8 CCDD. In Fig. 11, profiles of vacuum ion current density are compared with measurements at three background pressures, clearly exhibiting increased ion current density in the central core due to neutral ingestion and elevated current density on the periphery due to CEX collisions with facility neutrals.

Current density profiles of the four nested Faraday probe configurations were extrapolated to vacuum conditions for all downstream distances in Fig. 12. These vacuum current density profiles provide insight into the ion migration that would be present in orbit. The configurations exhibit consistent profiles at all distances and further increase confidence in the methods developed for determination of vacuum current density. The current density of configuration 1 was slightly larger than the other configurations, and it was attributed to measurement and/or alignment errors of the inner collector that resulted in miscalculation of the ion collection area. This increased current density profile manifested as a $\sim 5 \%$ increase in the integrated ion beam current compared with the other configurations. The thruster current utilization efficiency, calculated as the ion beam current relative to the total thruster discharge current $I_{d}$, is listed in Table 2 for vacuum conditions of all probe configurations at all downstream distances. Configurations 2,3 , and 4 were within a 0.03 range of current utilization for all downstream distances, and the magnitudes of ion beam current relative to discharge current were
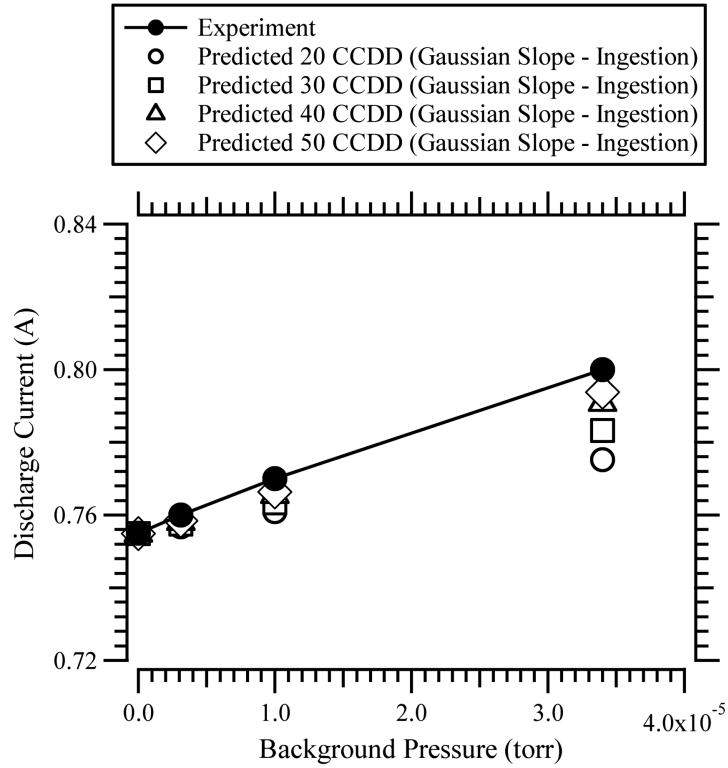

Fig. 10 Experimental discharge current and predicted discharge current based on the Gaussian function for ingestion of facility neutral particles as a function of background pressure. Predicted discharge currents are shown for 8, 12, 16, and 20 CCDD. consistent with values expected from analysis of Hall thruster performance [19].

\section{Discussion}

\section{A. Ion Migration in a Hall Thruster Plume}

Elimination of particle scattering generated by facility effects enabled the study of beam divergence and ion migration in the plume. Figure 13 shows profiles of vacuum current density per unit solid angle at $8,12,16$, and 20 CCDD. The residuals reveal the angular regions where the slope was approximately zero and background pressure had a minimal effect on the plume.

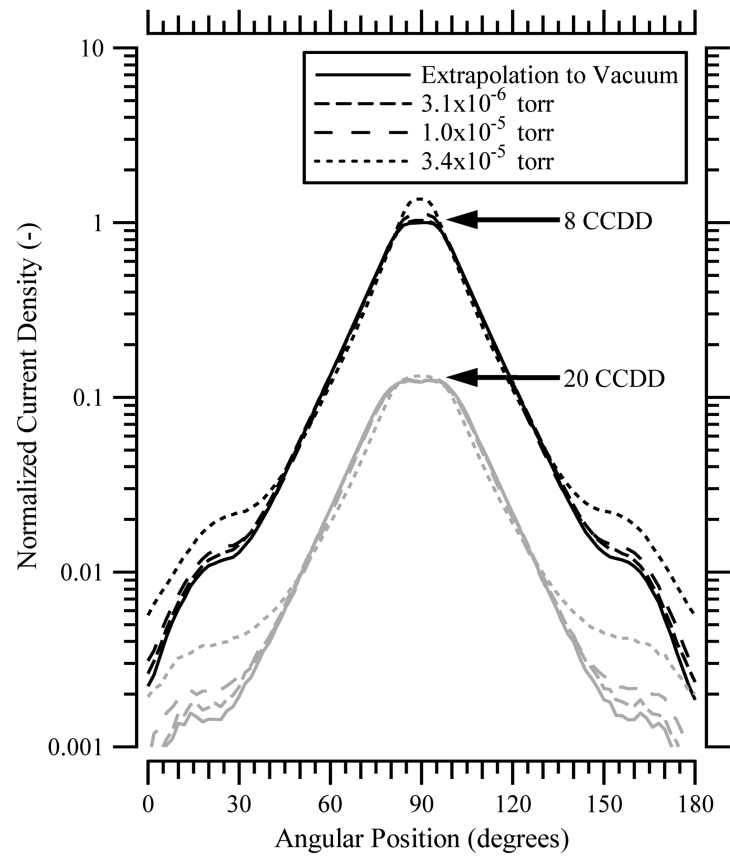

Fig. 11 Normalized ion current density profiles of nested Faraday probe configuration 1 as a function of angular position at 8 and 20 CCDD.

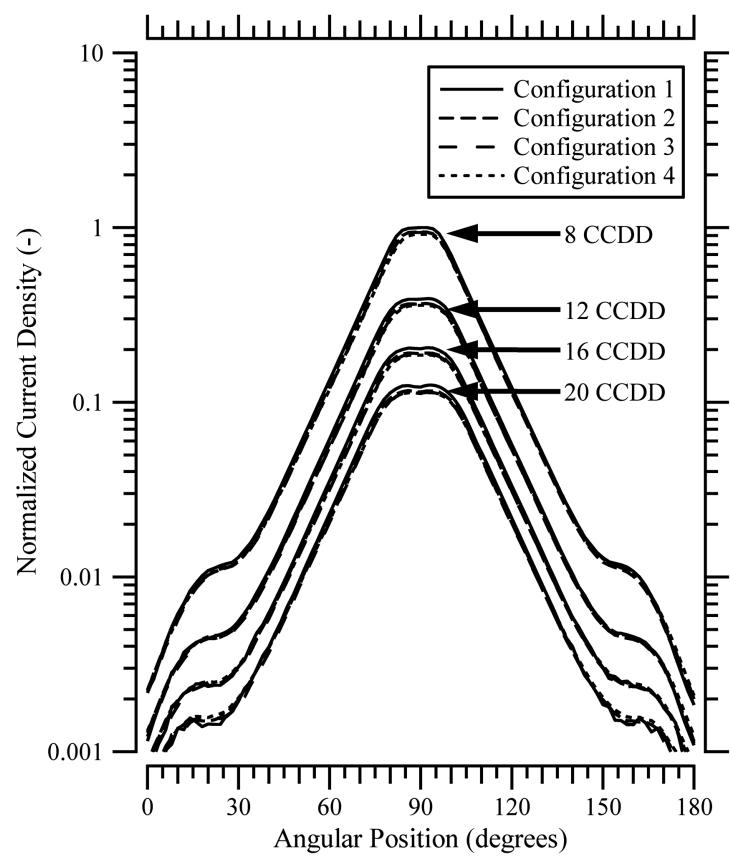

Fig. 12 Normalized ion current density profiles at vacuum conditions for nested Faraday probe configurations 1, 2, 3, and 4 as a function of angular position. 
Table 2 Ratio of integrated ion beam current to thruster discharge current at vacuum conditions for nested Faraday probe configurations 1 to 4 at 8, 12, 16, and 20 CCDD

\begin{tabular}{ccccc}
\hline \hline & \multicolumn{4}{c}{$I_{\text {Beam }} / I_{d}$} \\
\cline { 2 - 5 } $\begin{array}{c}\text { Downstream } \\
\text { distance, CCDD }\end{array}$ & Configuration 1 & Configuration 2 & Configuration 3 & Configuration 4 \\
\hline 8 & 0.88 & 0.82 & 0.81 & 0.80 \\
12 & 0.89 & 0.84 & 0.82 & 0.81 \\
16 & 0.90 & 0.84 & 0.82 & 0.82 \\
20 & 0.90 & 0.85 & 0.82 & 0.83 \\
\hline \hline
\end{tabular}

Total ion migration in the plume was studied in greater detail using the angular distribution of ion beam current through the surface of a spherical stripe, as illustrated in Fig. 14. The angular distribution of ion beam current passing through a constant angular width stripe $\mathrm{d} \theta$ allowed spatial analysis of beam current transport with distance and angle:

$$
I_{\text {Stripe }}[\theta, R]=2 \pi R^{2} \frac{I[\theta, R]}{A_{C}+\kappa_{G}}\left(\frac{\kappa_{D}\left[\theta, R, R_{\mathrm{CL}}\right]}{\kappa_{A}\left[\theta, R, R_{\mathrm{CL}}\right]}\right) \sin [\theta+(\pi / 2)] \mathrm{d} \theta
$$

and

$$
\sum_{0}^{\theta=\pi / 2} I_{\text {Stripe }}[\theta]=I_{\text {Beam }}
$$

This calculation was based on the integral for total ion beam current in Eq. (6). The sum of ion beam current per unit stripe passing through a half-angle resulted in the ion beam current per steradian, and the sum from $\theta=0$ to $90^{\circ}$ was the total ion beam current $I_{\text {Beam }}$. The ion beam current passing through a stripe will be referred to in units of amperes per $1^{\circ}$ unit stripe (A/unit stripe).

The trends in Fig. 11 are examined using angular distributions of ion beam current per unit stripe in Figs. 15-17, where all profiles are normalized to the maximum ion beam current per unit stripe at vacuum conditions and $8 \mathrm{CCDD}$. The angular distribution of vacuum ion beam current per unit stripe is shown for 8 CCDD in Fig. 15. Figure 16 exhibits the differences in ion beam current at $3.1 \times 10^{-6}$, $1.0 \times 10^{-5}$, and $3.4 \times 10^{-5}$ torr relative to the vacuum ion beam

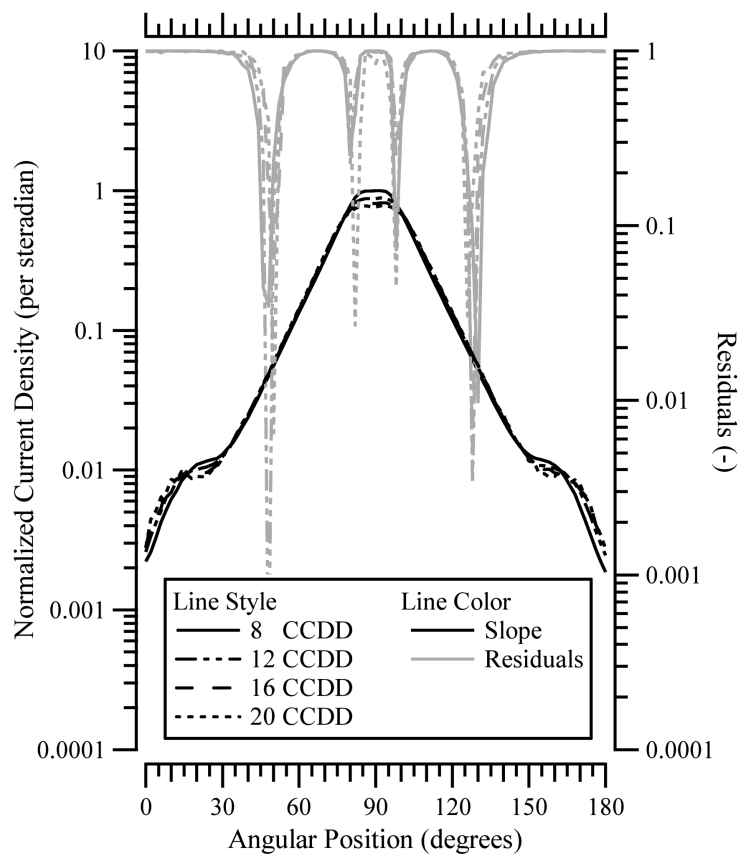

Fig. 13 Normalized profiles of ion current density per steradian at vacuum conditions and residuals of nested Faraday probe configuration 1 as a function of angular position. current profile in Fig. 15, calculated as $I_{\text {Stripe }}[\theta, p]-I_{\text {Stripe }}[\theta, p=$ vacuum] at $8 \mathrm{CCDD}$. These trends highlight the influence of facility effects on the current density profiles relative to the vacuum conditions. Elevated facility background pressure amplified the ion beam current on the periphery and increased ion beam current in the central core due to ingested facility neutrals. In this study, deviations from the vacuum beam current distribution reached $20 \%$ near the central core at 20 CCDD and the highest background pressure.

Figure 17 shows the differences in vacuum ion beam current at 12,16 , and 20 CCDD relative to the vacuum ion beam current profile at $8 \mathrm{CCDD}$ in Fig. 15 , calculated as $I_{\text {Stripe }}[\theta, R]-I_{\text {Stripe }}[\theta, R=$ $8 \mathrm{CCDD}]$. The relative difference in the vacuum ion beam current per unit stripe in Fig. 17 was negligible at six angular locations in the plume for all downstream distances, not including the outer periphery at $\theta=0$ and $180^{\circ}$. Regions of constant ion beam current per unit stripe were located at $\theta=16,34$, and $78^{\circ}$ on the left side of the plume and $\theta=101,146$, and $163^{\circ}$ on the right side of the plume.

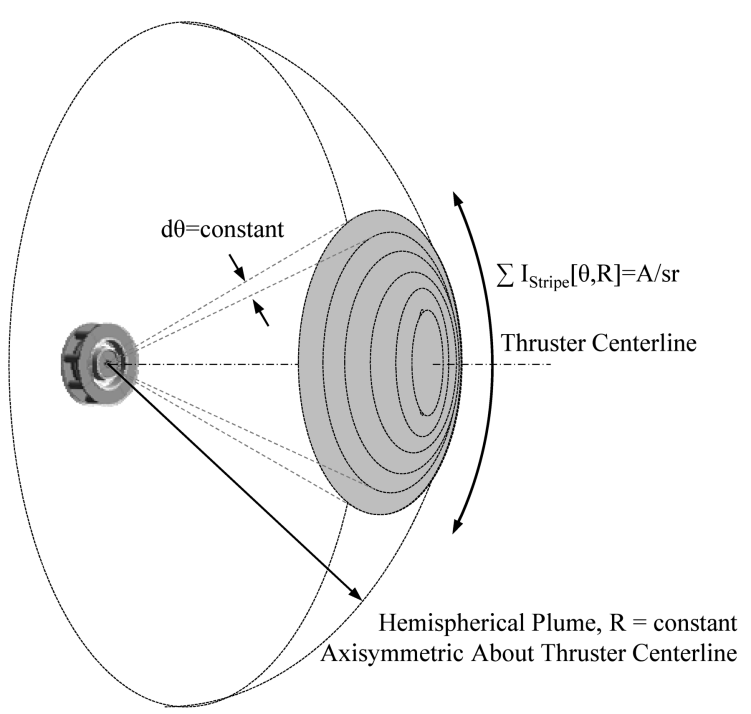

Fig. 14 Diagram of axisymmetric spherical stripe coordinate geometry for analysis of angular ion beam current distribution in the plume.

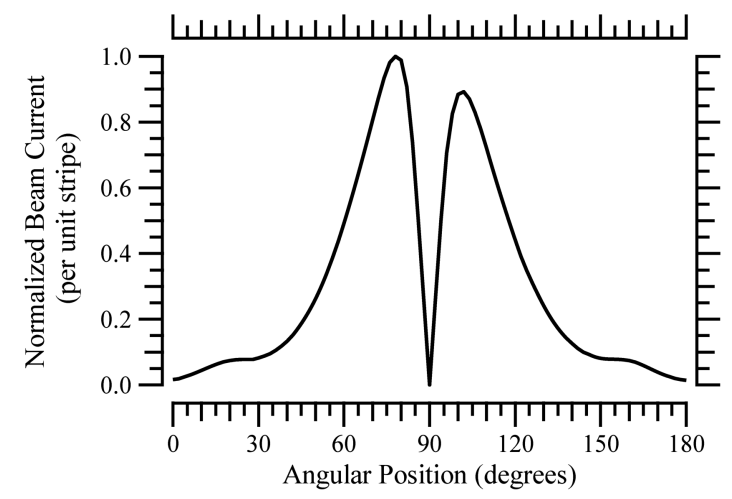

Fig. 15 Normalized ion beam current per unit stripe at vacuum conditions and 8 CCDD. 


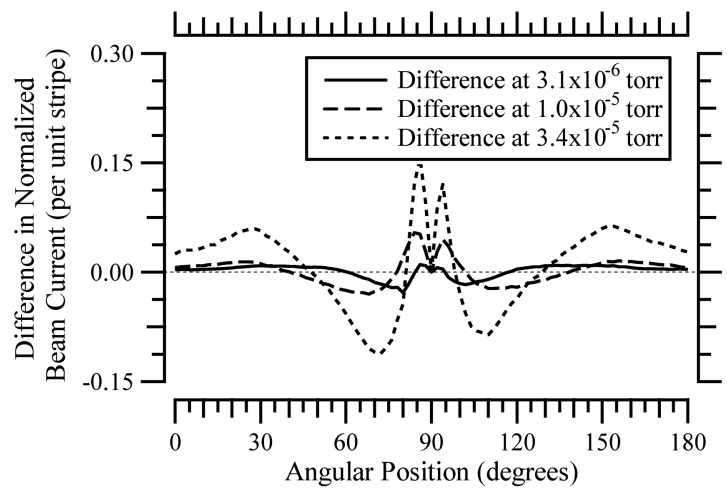

Fig. 16 Difference between normalized ion beam current per unit stripe at 8 CCDD and elevated background pressure with respect to the profile at vacuum conditions.

These locations were consistent to within $\pm 1^{\circ}$ at all distances, and they corresponded to the zones where the residuals decreased and $\tau$ was approximately zero in Fig. 8. The differences in vacuum ion beam current per unit stripe relative to the profile at $8 \mathrm{CCDD}$ in Fig. 17 were studied from $\theta=0^{\circ}$ to $\theta=90^{\circ}$ to evaluate macroscopic ion migration in the plume. In Fig. 18, the outward migration of ion current per unit stripe was examined as a function of angular position to evaluate the total transfer of beam current per steradian $\Delta I_{\text {Stripe }}$ from the central core at $\theta=90^{\circ}$ to the periphery of the plume at $\theta=0^{\circ}$ :

$$
\Delta I_{\text {Stripe }}[\theta, R]=\sum_{\theta}^{\theta=\pi / 2} I_{\text {Stripe }}[\theta, R]-\sum_{\theta}^{\theta=\pi / 2} I_{\text {Stripe }}[\theta, R=8 \text { CCDD }]
$$

Figure 18 reveals the overall transfer of ion beam current from 78 to $90^{\circ}$ was transferred to the region between 34 and $78^{\circ}$, such that $\Delta I_{\text {Stripe }} \approx 0$ at $\theta=34^{\circ}$. A negligible fraction of current migrated beyond $34^{\circ}$, and it implied that the primary ion beam was conserved within this angle for vacuum conditions at all downstream distances in the far-field plume. In a similar manner to the primary beam, the ion current from 16 to $34^{\circ}$ was transferred to the region between 0 and $16^{\circ}$ at all downstream distances. The integrated vacuum ion beam current in the plume from 0 to $180^{\circ}$ varied by less than $2 \%$ for all downstream locations in Fig. 18.

The experimental ion migration trends for vacuum conditions in Fig. 17 were compared with preliminary numerical simulations of the far-field plume using DRACO [20], an electrostatic PIC module in COLISEUM $[21,22]$. DRACO uses a hybrid-PIC model of plasma processes with a kinetic description of heavy particles and a fluid description for the electrons. Collisions were modeled with Monte Carlo collision (MCC) methods [23] in a Cartesian mesh

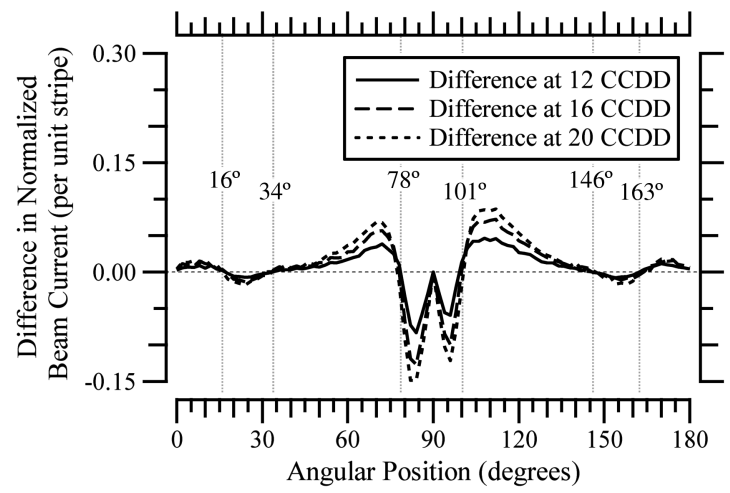

Fig. 17 Difference in normalized vacuum ion beam current per unit stripe at 12, 16, and $20 \mathrm{CCDD}$ with respect to the vacuum profile at 8 CCDD.

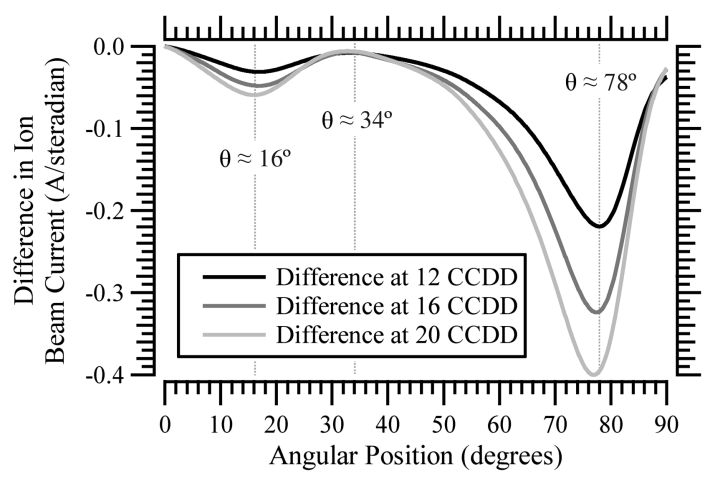

Fig. 18 Difference in vacuum ion beam current per steradian at 12, 16, and $20 \mathrm{CCDD}$ relative to the vacuum profile at $8 \mathrm{CCDD}$, evaluated as a function of angular position from the central core at $\theta=90^{\circ}$ to the periphery of the plume at $\theta=0^{\circ}$.

framework. DRACO supports the standard finite difference PIC method and uses a Boltzmann factor to relate electron density to the electric potential [24].

In these numerical simulations of the far-field plume, the ion source model was determined with a modified version of HPHall-2 using a three-region mobility model [25]. HPHall-2 is an axisymmetric, hybrid fluid/PIC model of the Hall thruster discharge, where heavy particles are modeled with PIC methods [26] and electrons are modeled as a fluid [27].

In this comparison of ion migration, elastic processes and CEX collisions with the background gas were not included in COLISEUM simulations of the far-field plume; therefore, the numerical simulations were compared with the experimental vacuum profiles. Two simulations of far-field plume expansion were performed. The first simulation included CEX collisions of beam ions with thruster neutrals. The second simulation did not incorporate CEX collisions between these populations. Comparisons of the experimental and simulated differences in vacuum ion beam current profiles relative to 8 CCDD are shown in Fig. 19, where all profiles were normalized to the maximum ion beam current per unit stripe at vacuum conditions and 8 CCDD. Differences in the potential field between the HPHall-2 ion source model and physical experiment are believed to be the cause of differences in the angular distributions of ion beam current.

The comparison of experimental and simulated results in Fig. 19 reveals key details about Hall thruster plume expansion and characteristics of ion migration in vacuum. The simulation including CEX collisions with thruster neutrals exhibited minimal difference

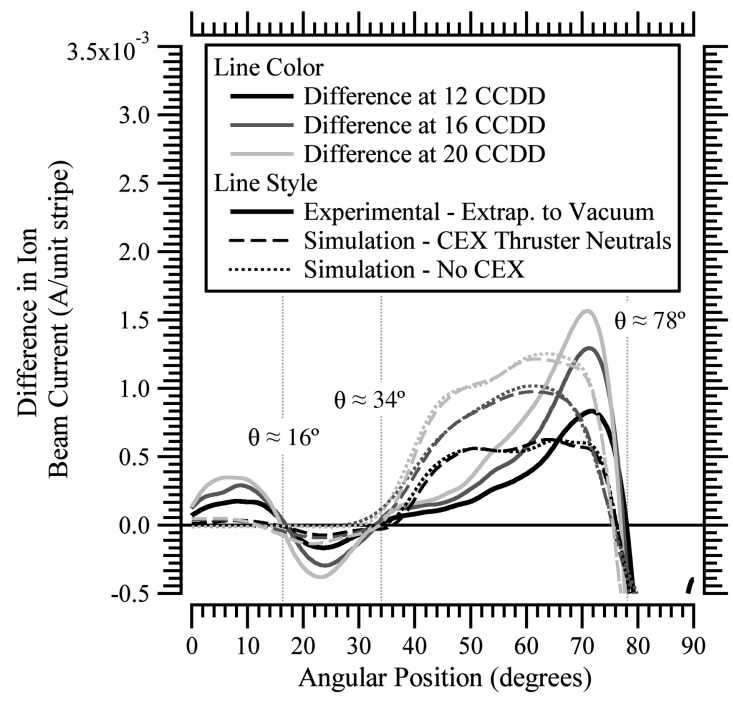

Fig. 19 Comparison of simulated and experimental differences in normalized vacuum ion beam current per unit stripe at 12, 16, and $20 \mathrm{CCDD}$ with respect to the vacuum profile at $8 \mathrm{CCDD}$. 
from the simulation without CEX processes. The simulated results matched the experimental regions of minimal ion migration near $\theta=34^{\circ}$ and $\theta=78^{\circ}$. In the absence of CEX collisions and elastic processes in the plume, a principal source of ion migration was due to gradients in the potential field external to the primary acceleration region. This indicated far-field angular regions of constant vacuum ion beam current per stripe $\left(\theta=90^{\circ}\right.$ to $\left.\theta=34^{\circ}\right)$ may arise due to minimal gradients in the external field, and far-field CEX collisions with thruster neutrals play a lesser role within this half-angle. In this case, the characteristics of ion migration in the far-field plume were captured with the COLISEUM model, despite probable differences in the near-field potential field between the source model and experiment. This outcome was attributed to negligible plasma potential gradients in the far-field plume compared with gradients in the near-field thruster plasma.

The simulation with thruster ion-neutral CEX collisions recreated features of the experimental ion beam current migration between $\theta=0^{\circ}$ and $\theta=34^{\circ}$. The lack of agreement within this region in the simulation without CEX collisions indicated this process is likely related to CEX collisions with thruster neutrals. The mechanisms that result in the overall plume characteristics were not fully understood and require additional simulation and analysis.

\section{B. Evaluation of Ion Beam Divergence}

To accurately assess plume divergence, it was necessary to characterize the migration of primary beam ions in the plume due to gradients in the far-field plasma potential and CEX collisions with anode and cathode neutrals. Although this scattering would be present in orbit, beam divergence downstream of the cathode neutralization plane does not diminish thrust. Therefore, accounting for this divergence would cause an overprediction of plume divergence losses in the analysis of Hall thruster efficiency.

Jet momentum losses due to beam divergence are naturally expressed as a momentum-weighted average cosine [19]. Divergence of ion current in the plume is indicative of the loss in thrust due to offaxis ion velocity, and it is often used as an alternative for experimental characterization of performance losses due to plume divergence. The momentum-weighted average cosine $\langle\cos (\theta)\rangle_{m v}$ was approximated as the current-weighted average $\operatorname{cosine}\langle\cos (\theta)\rangle_{J}$ for an axisymmetric plume in Eq. (11):

$$
\langle\cos (\theta)\rangle_{m v} \cong \frac{2 \pi R^{2} \int_{0}^{\pi / 2} I[\theta, R] \cos (\theta) \sin (\theta) \mathrm{d} \theta}{2 \pi R^{2} \int_{0}^{\pi / 2} I[\theta, R] \sin (\theta) \mathrm{d} \theta}=\langle\cos (\theta)\rangle_{J}
$$

An effective plume divergence angle $\lambda$ was calculated as shown in Eq. (12):

$$
\lambda=\cos ^{-1}\left(\langle\cos (\theta)\rangle_{J}\right)=\cos ^{-1}\left(\frac{I_{\text {Axial }}}{I_{\text {Beam }}}\right)
$$

This angle was significantly less than the $95 \%$ divergence halfangle that is typically reported for evaluation of plume expansion in electric propulsion thrusters.

The axial component of ion beam current was studied relative to the angle from the channel centerline, as opposed to the conventional reference of the thruster centerline. Figure 20 illustrates the reduction in plume divergence half-angle with respect to the channel centerline $\alpha_{A}$ compared with the plume divergence angle with respect to the thruster centerline $\left(90^{\circ}-\theta\right)$. The reference frame based on channel centerline reduced systematic error in plume divergence associated with beam ions in the central core, and it was similar to the methodology developed in Sec. III to reduce the systematic error of a point source measurement coordinate system. The axial component of ion beam current $I_{\text {Axial }}$ was calculated with respect to the channel centerline using $\alpha_{A}$ in Eq. (13):

$I_{\text {Axial }}=2 \pi R^{2} \int_{0}^{\pi / 2} \frac{I[\theta, R] \cos \left(\alpha_{A}\left[\theta, R, R_{\mathrm{CL}}\right]\right)}{A_{C}+\kappa_{G}}\left(\frac{\kappa_{D}\left[\theta, R, R_{\mathrm{CL}}\right]}{\kappa_{A}\left[\theta, R, R_{\mathrm{CL}}\right]}\right) \sin (\theta) \mathrm{d} \theta$

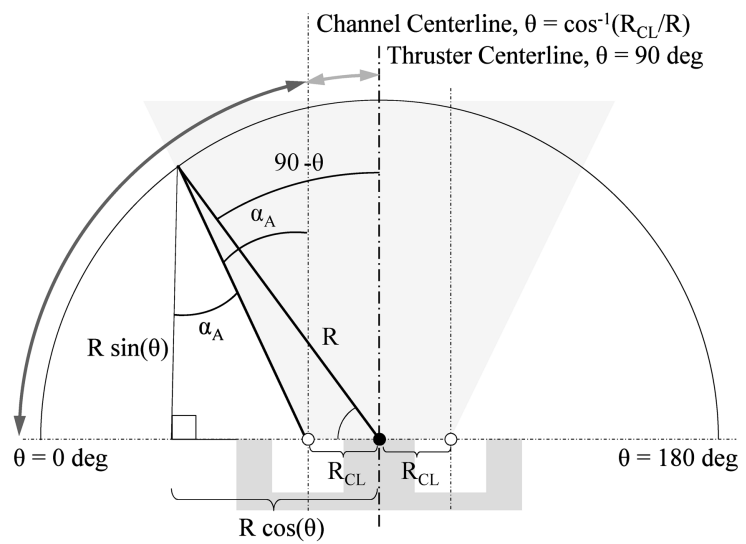

Fig. 20 Diagram of the axial component of beam current relative to channel centerline.

In Fig. 20, the cosine loss in beam current was fixed at $\alpha_{A}=0^{\circ}$ in the central core and calculated with respect to the channel centerline in the region beyond the central core out to $\theta=0^{\circ}$. This piecewise function for $\alpha_{A}$ is expressed in Eq. (14):

$$
\begin{aligned}
& \alpha_{A}\left[\theta, R, R_{\mathrm{CL}}\right] \\
& \quad= \begin{cases}\tan ^{-1}\left(\frac{\cos (\theta)-\left(R_{\mathrm{CL}} / R\right)}{\sin (\theta)}\right) & \text { for } 0^{\circ} \leq \theta \leq \cos ^{-1}\left(\frac{R_{\mathrm{CL}}}{R}\right) \\
0 & \text { for } \cos ^{-1}\left(\frac{R_{\mathrm{CL}}}{R}\right) \leq \theta \leq 90^{\circ}\end{cases}
\end{aligned}
$$

The ratio of the axial component of ion beam current calculated in Eq. (13) relative to the total ion beam current determined from Eq. (6) is shown for all background pressures and all configurations of the nested Faraday probe in Fig. 21. As downstream distance increased, the ratio decreased for all cases. This effect was expected and attributed to divergence caused by the plasma potential gradients in the plume, CEX collisions with anode and cathode neutrals, and CEX collisions with facility neutrals for the profiles at finite background pressure.

Experimental data were fit with a second-order polynomial trendline to determine an effective divergence angle near the thruster exit plane. All 16 trendlines of the ratio $I_{\text {Axial }} / I_{\text {Beam }}$ converged to $0.94 \pm 0.01$, corresponding to a divergence angle of approximately $\lambda=20^{\circ}$. This precision over a wide range in background pressure, measurement distance, and probe geometry was a significant milestone for determination of cosine losses in Hall thruster performance.

It should be noted that the ratio of $I_{\text {Axial }} / I_{\text {Beam }}$ extrapolated to vacuum conditions showed a significant decline with downstream distance, albeit less than the reduction with finite facility background pressure. This indicated a significant source of plume divergence downstream of the thruster exit was unrelated to facility effects. Based on these measurements, the ratio of $I_{\text {Axial }} / I_{\text {Beam }}$ may diminish by more than $5 \%$ in the near-field plume before reaching a steady value in the far-field plume. This divergence occurred predominantly beyond the primary beam ion acceleration region, and it would have a negligible effect on the directed thrust or performance [28].

The coefficients of the second-order polynomial trendlines varied with background pressure. No universal function was found that correlated these coefficients to background pressure, discharge voltage, and mass flow rate. For a general second-order polynomial expression of the form $y(x)=A_{2} x^{2}+A_{1} x+A_{0}$, increased background pressure increased the coefficient $A_{1}$ and decreased the coefficient $A_{2}$. In this formulation, the coefficient $A_{0}$ was the ratio $I_{\text {Axial }} / I_{\text {Beam }}$ at vacuum conditions.

Variations in the polynomial coefficients with discharge voltage and anode mass flow rate were more difficult to isolate and quantify due to the dependence on plume focusing and the location of 


$$
\begin{aligned}
& \longrightarrow \text { - Extrapolation to Vacuum } \\
& \text { …............ } 3.1 \times 10^{-6} \text { torr } \\
& ---0--1.0 \times 10^{-5} \text { torr } \\
& --0--3.4 \times 10^{-5} \text { torr }
\end{aligned}
$$

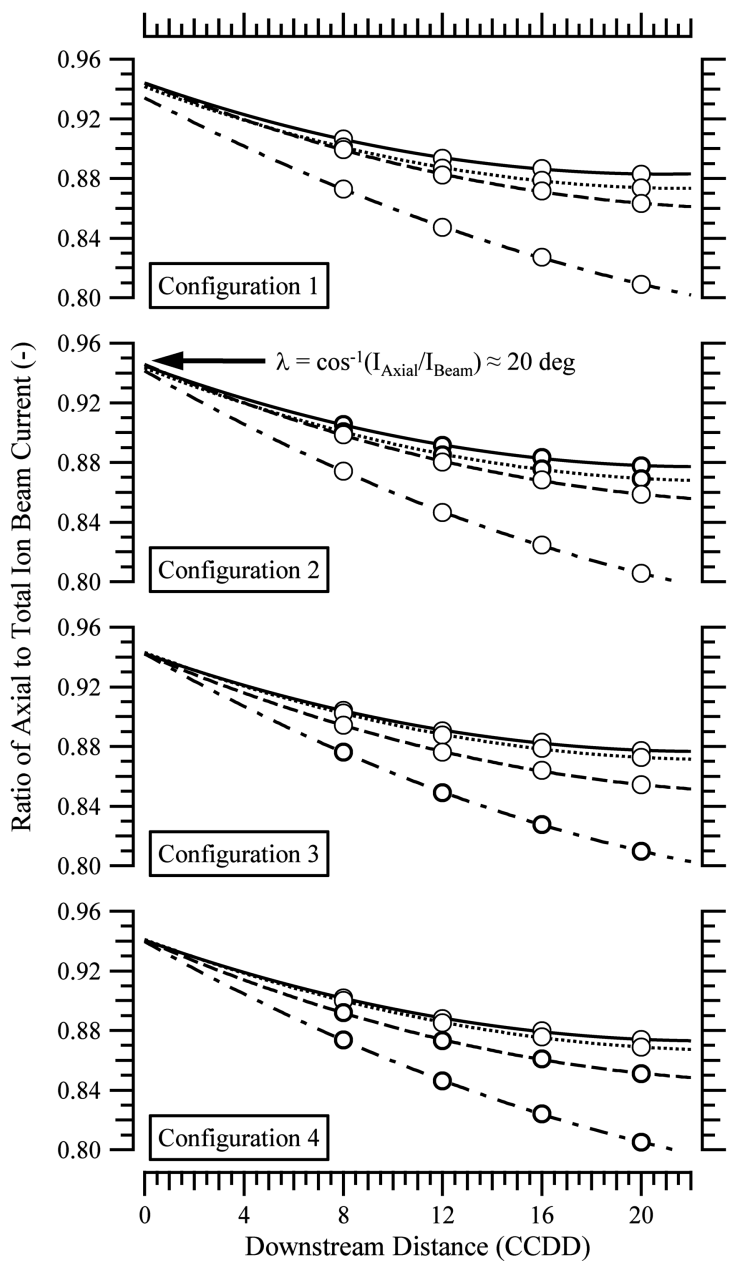

Fig. 21 Experimental data (markers) and second-order polynomial trendlines of the ratio of the axial component of ion beam current relative to the total ion beam current.

ionization. This limited data set indicated higher thruster discharge voltage decreased the magnitude of coefficients $A_{1}$ and $A_{2}$. The relationship was attributed to the more collimated beam that was associated with high discharge voltage operation.

Anode mass flow rate was believed to have two competing effects on divergence. Increased propellant flow rate corresponded to a narrower axial region of ionization and acceleration in the discharge, along with a more concentrated ion density near channel centerline [29]. These effects led to a decrease in divergence due to enhanced plume focusing, and they would likely have a similar effect as discharge voltage on polynomial coefficients. However, the increased neutral flow may also lead to increased CEX collisions with thruster neutrals downstream of the exit plane, thereby increasing ion scattering in the far-field plume. A simple analytical model was deemed insufficient to fully characterize the influence of beam focusing and facility effects on plume divergence. Additional systematic investigations and numerical simulations with a high-fidelity source model are required to determine these relationships.

\section{Recommendations for High-Accuracy Faraday Probe Current Density Measurements}

Analytical methods and experimental techniques described in this paper may be used to determine Hall thruster current density profiles and integrated ion beam current to a high degree of accuracy. To minimize uncertainty of far-field Faraday probe measurements, the following guidelines are recommended for Faraday probe design, experimental approach, and analysis of results. Several of these guidelines are conventional practice or have been recommended in previous literature $[2,9,13]$.

1) Select a Faraday probe design with a 5 to 10 Debye length gap between the collector and guard ring in order to measure plasma properties over a wide range of downstream distances and pressures. Select collector and guard ring material with minimal SEE coefficient, such as molybdenum, graphite, or tungsten [2].

2) Conduct Faraday probe current density measurements at a minimum of three facility background pressures to determine the vacuum current density profiles for calculation of total ion beam current. Measurements at additional pressures will reduce uncertainty of vacuum ion current density distributions. The background pressures should range by at least one order of magnitude.

3) Conduct Faraday probe current density measurements at a minimum of four downstream distances to determine the axial component of ion beam current at the exit plane and calculate ion beam divergence. For far-field measurements about a single axis of rotation using a spherical measurement coordinate system, the distances should be greater than 8 CCDD. For near-field measurements based on a cylindrical measurement coordinate system, spatial effects and cosine losses should be estimated and the maximum distance should be less than approximately one thruster diameter (1 CCDD) downstream using a dynamic window integration method [29] or similar technique. Measurements between 1 and 8 CCDD will have increased uncertainty due to the measurement coordinate system.

4) Include the correction factors $\kappa_{A}, \kappa_{D}$, and $\alpha_{A}$ to account for the use of a point source measurement coordinate system geometry for an annular thruster device.

5) Account for ions collected in the gap between the collector and guard ring by increasing the effective ion collection area with $\kappa_{G}[14]$.

6) Consider effects of ion collection at the base of the gap between the collector and guard ring when selecting a Faraday probe design. A ceramic base is recommended for investigations at variable or high background pressure [14].

The guidelines provide a framework for determination of onorbit current density profiles and minimize experimental measurement uncertainty. Based on the precision of results in this investigation and consistent agreement between multiple probes over a range of farfield distances and facility background pressures, the recommendations are expected to reduce uncertainty of total ion beam current to $\pm 3 \%$ and reduce uncertainty of the axial component of ion beam current to $\pm 5 \%$. Accurate assessment of the loss in thrust due to ion beam divergence requires ion beam characterization with variations in downstream distance. Assessment of ion beam current requires characterization with variations in background pressure. Ideally, the experimental current density profiles extrapolated to vacuum will enable comparison of ground-based measurements with numerical simulations in the absence of facility effects, thereby reducing computational complexity and time.

Near-field measurements would seem to minimize the uncertainty associated with far-field measurements. However, these measurements introduce unique challenges, including probe-induced perturbation of the plasma discharge, a wider range of Debye length in the measurement domain, larger gradients in the plasma potential, and possibly SEE effects from the probe collector. In addition, ingestion and near-field CEX collisions with facility neutrals are also present in near-field measurements. Thus, the near-field ion current density profiles should also be characterized for variations in distance and background pressure. A second set of spatial corrections for measurement coordinate geometry may also be necessary to reduce systematic error associated with cylindrical integration as distance from the exit plane increases.

\section{Conclusions}

A comprehensive investigation of facility effects on Hall thruster current density measurements was studied over a range of down- 
stream measurement distances and background pressures with four configurations of a nested Faraday probe. The primary aim of this investigation was to isolate facility effects and thereby enable examination of ion migration in a Hall thruster plume in the absence of background neutral population. Geometric correction factors and methods for evaluating plume properties minimized systematic measurement error and characterized facility interactions on current density distributions. The corrected plume properties were in line with expected values of ion beam current based on Hall thruster performance and discharge properties. The integrated vacuum ion beam current measurements were within a $3 \%$ range for all nested Faraday probe configurations at all distances. This level of precision required measurements over a range of facility background pressure of approximately one order of magnitude.

Variations in the ion current density profiles as a function of facility background pressure were studied as the superposition of two Gaussian curves. These curves provided quantitative information about the ionization of ingested neutrals and CEX collisions with facility neutrals in the plume. The influence of neutral particle ingestion on measured ion current density decreased with downstream distance, but it affected a wider angular span in the extended far field. Scattering of primary beam ions increased at elevated facility pressure, and it affected a constant angular region of the plume. The results provide a means to assess facility interactions on ion migration in the plume, which could be used for identification of criteria for acceptable background pressure during ground tests.

Examination of the ratio of axial component of ion beam current relative to total ion beam current over the range of background pressure and distance indicated a significant source of plume divergence occurred downstream of the thruster exit, and it was unrelated to facility effects. This divergence occurred predominantly beyond the primary ion acceleration zone, and it would have a negligible effect on the net thrust. The far-field plume divergence was attributed to CEX collisions with thruster neutrals and divergence associated with gradients in the plasma potential, both of which are present in orbit. Thus, determination of beam divergence on thruster performance should either be accomplished with near-field measurements or through characterization of $I_{\text {Axial }} / I_{\text {Beam }}$ with downstream distance. The ratio was extrapolated to the thruster exit plane with a second-order polynomial, resulting in consistent agreement to within $1 \%$ for all background pressures and probe configurations. This agreement supported the experimental methods and provided an accurate representation of plume divergence losses in thruster efficiency. The recommended experimental methods and analytical techniques for determination of vacuum ion current density with Faraday probes offer a significant improvement in capability to study far-field plasma characteristics in a Hall thruster plume, and they are expected to aid future comparisons of ground-based measurements with computational simulations and onorbit data.

\section{Acknowledgments}

The authors would like to thank Michelle Scharfe for supplying simulated plume data, Justin Koo and the late Bill Larson for numerous insightful discussions, and Mike Nakles and Garrett Reed for assistance with the experimental setup and data acquisition.

\section{References}

[1] Manzella, D. H., and Sankovic, J. M., "Hall Thruster Ion Beam Characterization," AIAA Paper 1995-0292, 1995.

[2] Walker, M. L. R., Hofer, R. R., and Gallimore, A. D., "Ion Collection in Hall Thruster Plumes," Journal of Propulsion and Power, Vol. 22, No. 1, 2006, pp. 205-209. doi: $10.2514 / 1.11953$

[3] de Grys, K. H., Tilley, D. L., and Aadland, R. S., "BPT Hall Thruster Plume Characteristics," AIAA Paper 1999-2283, 1999.

[4] Bugrova, A. I., and Morozov, A. I., "Influence of Vacuum Conditions on the SPT Operation," Proceedings of the 24th International Electric Propulsion Conference, IEPC Paper 95-0046, 1995.

[5] Nakles, M. R., and Hargus, W. A., Jr., "Background Pressure Effects on Internal and Near-field Ion Velocity Distribution of the BHT-600 Hall Thruster," AIAA Paper 2008-5101, 2008.
[6] Manzella, D. H., Jankovsky, R., Elliott, F., Mikellides, I., Jongeward, G., and Allen, D., "Hall Thruster Plume Measurements On-Board the Russian Express Satellite," Proceedings of the 27th International Electric Propulsion Conference, IEPC Paper 01-044, 2001.

[7] Boyd, I. D., and Dressler, R. A., "Far Field Modeling of the Plasma Plume of a Hall Thruster," Journal of Applied Physics, Vol. 92, No. 4, 2002, pp. 1764-1774.

doi:10.1063/1.1492014

[8] Korsun, A. G., Tverdokhlebova, E. M., and Gabdullin, F. F., "The Distinction Between the EP Plume Expansion in Space and in Vacuum Chamber," Proceedings of the 29th International Electric Propulsion Conference, IEPC Paper 05-0073, 2005.

[9] Hofer, R. R., Walker, M. L. R., and Gallimore, A. D., "A Comparison of Nude and Collimated Faraday Probes for Use with Hall Thrusters," Proceedings of the 27th International Electric Propulsion Conference, IEPC Paper 01-020, 2001.

[10] Rovey, J. L., Walker, M. L. R., Gallimore, A. D., and Peterson, P. Y., "Magnetically Filtered Faraday Probe for Measuring the Ion Current Density Profile of a Hall Thruster," Review of Scientific Instruments, Vol. 77, No. 1, 2006, pp. 013503. doi:10.1063/1.2149006

[11] Walker, M. L. R., Victor, A. L., Hofer, R. R., and Gallimore, A. D., "Effect of Backpressure on Ion Current Density Measurements in Hall Thruster Plumes," Journal of Propulsion and Power, Vol. 21, No. 3 , 2005, pp. 408-415. doi: $10.2514 / 1.7713$

[12] Boerner, J. J., "Computational Simulation of Faraday Probe Measurements," Ph.D. Dissertation, Univ. of Michigan, Ann Arbor, MI, 2008.

[13] Azziz, Y., "Experimental and Theoretical Characterization of a Hall Thruster Plume," Ph.D. Dissertation, Massachusetts Inst. of Technology, Cambridge, MA, 2007.

[14] Brown, D. L., and Gallimore, A. D., "Evaluation of Ion Collection Area in Faraday Probes," Review of Scientific Instruments, Vol. 81, No. 6, 2010, Paper 063504. doi:10.1063/1.3449541

[15] "Bulletin 01/08: Series 943 Cold Cathode," MKS Instruments, Andover, MA, 24 Feb. 2010.

[16] Walker, M. L. R., and Gallimore, A. D., "Neutral Density Map of Hall Thruster Plume Expansion in a Vacuum Chamber," Review of Scientific Instruments, Vol. 76, No. 5, 2005, Paper 053509. doi:10.1063/1.1915011

[17] Redhead, P. A., "Instabilities in Crossed-Field Discharges at Low Pressures," Vacuum, Vol. 38, Nos. 8-10, 1988, pp. 901-906. doi:10.1016/0042-207X(88)90489-7

[18] Peacock, R. N., Peacock, N. T., and Hauschulz, D. S., "Comparison of Hot Cathode and Cold Cathode Ionization Gauges," Journal of Vacuum Science and Technology A (Vacuum, Surfaces, and Films), Vol. 9, No. 3, 1991, pp. 1977-1985. doi:10.1116/1.577439

[19] Brown, D. L., Larson, C. W., Beal, B. E., and Gallimore, A. D., "Methodology and Historical Perspective of a Hall Thruster Efficiency Analysis," Journal of Propulsion and Power, Vol. 25, No. 6, 2009, pp. $1163-1177$. doi:10.2514/1.38092

[20] Brieda, L., Pierru, J., Kafafy, R., and Wang, J., "Development of the DRACO Code for Modeling of Electric Propulsion Plume Interactions," AIAA Paper 2004-3633, 2004.

[21] Fife, J. M., and Gibbons, M. R., Hargus, W. A., Jr., VanGilder, D. B., Kirtley, D. E., and Johnson, L. K., "The Development of a Flexible, Usable Plasma Interaction Modeling System," AIAA Paper 2002-4267, 2002.

[22] Gibbons, M. R., Kirtley, D. E., Van Gilder, D. B., and Fife, J. M., "Flexible Three-Dimensional Modeling of Electric Thrusters in Vacuum Chambers," AIAA Paper 2003-4872, 2003.

[23] Birdsall, C. K., "Particle-in-Cell Charge-Particle Simulations, Plus Monte Carlo Collisions with Neutral Atoms, PIC-MCC," IEEE Transactions on Plasma Science, Vol. 19, No. 2, 1991, pp. 65-85. doi:10.1109/27.106800

[24] Spicer, R. L., "Validation of the DRACO Particle-in-Cell Code Using Busek 200-W Hall Thruster Experimental Data," M.S. Thesis, Virginia Polytechnic Inst. and State Univ., Blacksburg, VA, 2008.

[25] Hofer, R. R., Katz, I., Mikellides, I. G., Goebel, D. M., Jameson, K. K., Sullivan, R. M., and Johnson, L. K., "Efficacy of Electron Mobility Models in Hybrid-PIC Hall Thruster Simulations," AIAA Paper 20084924, 2008.

[26] Birdsall, C. K., and Langdon, A., Plasma Physics Via Computer Simulations, Inst. of Physics Publ., Philadelphia, 2000.

[27] Parra, F. I., Ahedo, E., Fife, J. M., and Martinez-Sanchez, M., "A TwoDimensional Hybrid Model of the Hall Thruster Discharge," Journal of 
Applied Physics, Vol. 100, No. 2, 2006, Paper 023304.

doi: $10.1063 / 1.2219165$

[28] Brown, D. L., "Investigation of Low Discharge Voltage Hall Thruster Characteristics and Evaluation of Loss Mechanisms," Ph.D. Dissertation, Univ. of Michigan, Ann Arbor, MI, 2009.

[29] Reid, B. M., "The Influence of Neutral Flow Rate in the Operation of
Hall Thrusters," Ph.D. Dissertation, Univ. of Michigan, Ann Arbor, MI, 2009.

J. Blandino Associate Editor 